Ann. Sci. Forest., 1977, 34 (3), 245-260.

Note technique

\title{
Développement des boutures racinées de chêne rouvre (Quercus petraea (M.) Liebl.). Premiers éléments d'une technique de production de plants
}

\author{
J. GARBAYE, B. KAZANDJIAN *, F. LE TACON \\ avec la collaboration technique de D. BOUCHARD, F. BOURGEOIS, G. COURRIER \\ Station de Recherches sur les Sols forestiers et la Fertilisation, \\ Centre national de Recherches forestières, I.N.R.A., \\ Champenoux 54280 Seichambs \\ * Stagiaire élève-ingénieur de l'ENITEF
}

\section{Résumé}

La multiplication végétative du chêne rouvre est possible par bouturage de pousses herbacées sous « mist ». Les boutures racinées obtenues présentent un système racinaire plagiotrope et une inhibition du développement de la partie aérienne après une phase d'arrêt de végétation.

Cette inhibition peut être levée par une période de quelques mois (durée minimale non déterminée) dans des conditions de croissance optimale : jours longs, température diurne supérieure à $20^{\circ} \mathrm{C}$ et alimentation par solution nutritive sur substrat de tourbe. Les plants peuvent ensuife terminer leur croissance en pépinière dans des conditions normales, et atteindre en un an une taille comparable à celle des plants issus de semis.

Une période de froid préalable à la phase de forçage n'est pas indispensable, mais améliore l'homogénéité de la reprise de croissance.

La morphologie des plants obtenus se rapproche d'autant plus de celle des semis (port de la partie aérienne, racines plongeantes) que la croissance est rapide.

\section{Introduction}

La technique de bouturage de pousses herbacées de chêne rouvre (Quercus petraea) permet d'obtenir de forts pourcentages d'enracinement, aussi bien à partir de jeunes plants qu'à partir de rejets de souche, permettant ainsi de multiplier des arbres adultes (Cornu, Garbaye et Le Tacon, 1975 et 1976).

Cependant, cette technique de multiplication végétative n'est applicable que si le développement des plants ainsi produits est satisfaisant (croissance, port, qualité de l'enr acinement, etc...).

Nos premières observations en 1974 et 1975 ayant montré une forte mortalité et une très faible croissance des boutures ayant hiverné en pépinière, nous avons 
réalisé en 1975-1976 une expérimentation portant sur les conditions de cultures des plants issus de boutures. Le principal but recherché était de lever l'inhibition de croissance qui se manifeste encore 2 ans après la mise en place des plants en pépinière.

\section{Matériel et Méthode}

Sept traitements portant sur la période novembre 1975-septembre 1976 ont été appliqués à un lot de boutures enracinées contenues dans des «paper-pots» de $7,5 \times 15$ centimètres remplis de tourbe pure (conditionnement sous lequel s'est effectué le bouturage, pendant l'été 1976). Ce lot regroupait, après randomisation, des boutures provenant de matériels différents génétiquement ou physiologiquement (rejets de souche, jeunes plants) et ayant subi différentes conditions de bouturage. Tout le matériel appartient à la même provenance géographique : forêt de Champenoux (Meurthe-et-Moselle). Chaque traitement comportait, au départ, de 50 à 100 boutures regroupées dans des bacs en matière plastique perforés pour facilite les manutentions. Les traitements sont schématisés dans la figure 1.

\section{Traitement $A$}

De novembre 1975 à mai 1976, les boutures dans leurs mottes sont placées à l'extérieur et soumises aux conditions climatiques hivernales de Nancy (gel, neige). Le minimum absolu enregistré sous abri à la pépinière a été de $-14,9{ }^{\circ} \mathrm{C}$. Début mai, les « paper-pots » sont retirés et les mottes sont repiquées à la densité de 25 par mètre carré dans une bâche de pépinière contenant de la tourbe fertilisée sur 40 centimètres de profondeur (fertilisation optimale pour la croissance de semis de chêne : Delran, Garbaye, Le Tacon, 1975).

\section{Traitement $B_{1}$}

De novembre à mai, les boutures en motte sont placées dans un local faiblement éclairé non chauffé (la température n'est descendue qu'une fois au-dessous de $0{ }^{\circ} \mathrm{C}$ ). En mai, elles sont repiquées en pépinières dans les mêmes conditions que pour le traitement $\mathrm{A}$.

\section{Traitement $B_{2}$}

Même hivernage que $B_{1}$, mais en mai repiquage en motte (après avoir retiré le « paper-pot») dans des pots de 5 litres contenant de la tourbe, arrosés à l'aide d'une solution nutritive assurant une bonne croissance des semis de chêne (Coïc, 1973) et placés en serre climatisée (jours longs de 16 heures, $20^{\circ} \mathrm{C}$ de jour et 10 à $12{ }^{\circ} \mathrm{C}$ de nuit).

\section{Traitement $C_{1}$}

De novembre à janvier, les boutures en mottes sont placées en chambre froide obscure à $+4{ }^{\circ} \mathrm{C}$. En janvier, elles sont repiquées en pots dans les mêmes conditions qu'en $B_{2}$ et elles restent dans la serre jours longs $+20^{\circ} \mathrm{C}$, solution nutritive jusqu'en mai ; elles sont alors repiquées en pépinière comme en $A$ ef $B_{1}$, avec la motte de tourbe contenue dans le pot. 


\section{Traitement $C_{2}$}

Comme en $C_{1}$ jusqu'en mai, mais les boutures restent en serre jours longs $+20^{\circ} \mathrm{C}$, solution nutritive jusqu'à la fin.

\section{Traitement $D_{1}$}

De novembre 1975 à mai 1976, les boutures restent en serre chauffée en jours longs, d'abord en « paper-pots » (novembre-janvier) puis en pots + solution nutritive (janvier-mai). Elles sont ensuite repiquées en pépinière.

\section{Traitement $\mathrm{D}_{2}$}

Comme en $D_{1}$, mais les boutures restent dans la serre jusqu'en septembre.

En plus de ces 7 traitements portant sur des boutures de 1975, nous avons expérimenté 2 traitements à partir de boutures produites en hiver (forçage de pieds mères en serre, bouturage de février à avril 1976) :

\section{Traitement $E_{1}$}

Boutures repiquées en motte en pépinière en mai, sur tourbe fertilisée.

\section{Traitement $E_{2}$}

Boutures repiquées dans des pots de 5 litres en mai, restant ensuite dans la serre chauffée en jours longs, avec solution nutritive.

\section{Remarques concernant les traitements}

- Tous les repiquages ont été effectués en motte pour minimiser la crise de transplantation.

- Pour des raisons de commodité (tri, manutention) toutes nos expériences ont été réalisées en containers («paper-pots», pots). Nous ne nous sommes pas préoccupés des déformations racinaires, qui seront bien sûr à éviter dans la pratique.

- Le plan de l'expérience n'est pas factoriel, du fait de la disponibilité limitée en boutures racinées fin 1975. On ne peut donc pas comparer tous les traitements entre eux (par exemple, hivernage à l'extérieur et à $+4^{\circ} \mathrm{C}$ : les durées sont différentes).

- Pour la même raison, les traitements ne sont pas répétés, et une interprétation statistique des taux de survie est impossible. Nous ne pourrons donc interpréter que des différences très marquées. Par contre, une comparaison de moyennes 2 à 2 a été faite sur la hauteur des plants issus de boutures.

\section{Résultats qualitatifs}

Phénologie de la reprise de croissance

Lors du bouturage proprement dit, avec brumisation, une partie seulement des boutures développent une petite pousse (Cornu, Garbaye, Le Tacon, 1976). 
Après l'arrêt de la brumisation (août-septembre), l'apparition des pousses se généralise, mais les jours raccourcissant, les boutures cessent de croître et perdent plus ou moins de fevilles.

A l'exception des traitements $E_{1}$ ef $E_{2}$, où la pousse ne s'arrête jamais, notre expérience porte donc sur des boutures en arrêt de végétation en novembre 1975 (dans l'état actuel de nos observations, nous ne parlerons ni de « dormance», ni « d'aoûtement »).

Traitements $A$ et $B_{1}$

Le débourrement a lieu fin avril, comme pour des semis ayant hiverné à l'extérieur. Cependant, la pousse est très faible et la mortalité des plants forte, malgré les bonnes conditions de croissance pour des semis (photo 1). Cette pousse est anormale : petites feuilles en rosette, puis $2 \mathrm{e}$ pousse très courte. Le port est buissonnant.

\section{Traitement $\mathrm{B}_{2}$}

Le débourrement a lieu normalement lors du repiquage en serre avec solution nutritive, en mai. Les plants ont fait de 2 à 3 pousses pendant l'été.

Traitements $C_{1}$ et $C_{2}$

Le débourrement a lieu dans le mois qui suit la mise en place des boutures en serre + solution nutritive (janvier), avec un étalement tout à fait normal pour une population de chêne. Une à 2 pousses normales se développent avant le mois de mai. Une à 2 pousses apparaissent ensuite, plus longues dans $C_{2}$ que dans $C_{1}$.

Traitements $D_{1}$ et $D_{2}$

Malgré leur mise en conditions de croissance dès novembre, les boutures ne débourrent pas avant fin janvier, et de façon beaucoup moins synchrone que dans les traitements $C_{1}$ et $C_{2}$. De plus, la première pousse est le plus souvent anormale : rosette de feuilles à lobes anormalement aigus, aspect chlorotique malgré les bonnes conditions de nutrition minérale.

Le développement redevient ensuite normal avec la $2^{\mathrm{e}}$ pousse et se poursuit comme en $\mathrm{C}_{1}$ et $\mathrm{C}_{2}$, avec 2 à 4 pousses au total.

Ces observations phénologiques montrent chez la jeune bouture racinée en arrêt de végétation une inhibition de la croissance que l'on peut lever par des conditions de climat et de nutrition minérale adaptées. Cette levée d'inhibition est plus rapide et plus homogène dans le temps si une période de froid précède la mise en serre chauffée en jours longs et dans de bonnes conditions de nutrition (solution nutritive). 


\section{Résultats quantitatifs}

Pour les 9 traitements, nous avons représenté sous forme schématique (fig. 1) le pourcentage de boutures vivantes début mai et fin septembre 1976 (largeur de la bande représentative de chaque traitement), la hauteur moyenne et le diamètre moyen au collet fin septembre 1976. Le tableau no 1 donne les résultats des comparaisons de moyennes sur les boutures des traitements pris 2 à 2 .

\section{TABLEAU 1}

Résultats des comparaisons de moyennes sur les hauteurs des traitements pris deux à deux. Une astérisque signifie que la différence est significative au risque de 5 p. 100 (test « $f$ »). Les traifements sont classés par ordre de hauteur décroissante.

Results of the comparisons of mean heights of treatments. An asterisk is for a significant difference at the 5 p. 100 level («t» test). Treatments are ranked according to decreasing height.

\begin{tabular}{|c|c|c|c|c|c|c|c|c|c|}
\hline Traitement & $\mathrm{D}_{2}$ & $E_{2}$ & $C_{2}$ & $\mathrm{~B}_{2}$ & $C_{1}$ & $\mathrm{D}_{1}$ & $E_{1}$ & $\mathrm{~B}_{1}$ & A \\
\hline $\mathrm{D}_{2}$ & & $*$ & $*$ & $*$ & $*$ & $*$ & $*$ & $*$ & $*$ \\
\hline $\mathrm{E}_{2}$ & & & NS & $*$ & * & $*$ & $*$ & $*$ & $*$ \\
\hline $\mathrm{C}_{2}$ & & & & $*$ & $*$ & $*$ & $*$ & $*$ & $*$ \\
\hline$B_{2}$ & & & & & NS & NS & $*$ & $*$ & * \\
\hline $\mathrm{C}_{1}$ & & & & & & NS & $*$ & $*$ & $*$ \\
\hline$D_{1}$ & & & & & & & $*$ & $*$ & $*$ \\
\hline $\mathrm{E}_{1}$ & & & & & & & & NS & * \\
\hline $\mathrm{B}_{1}$ & & & & & & & & & NS \\
\hline$A$ & & & & & & & & & \\
\hline
\end{tabular}

D'une manière générale, on remarque que les plus mauvais résultats (tant pour le taux de survie que pour la taille des plants obtenus) correspondent aux traitement $A$ ef $B_{1}$, c'est-à-dire aux seules boutures ayant accompli toute leur croissance à l'extérieur sans jamais être passées par le stade solution nutritive + serre $20^{\circ} \mathrm{C}$ jours longs. La forte mortalité observée dans ces 2 traitements $A$ ef $B_{1}$ a eu lieu après le repiquage en pépinière de mai 1976, et non pas pendant la période d'hivernage proprement dite. Comme, d'autre part, la survie à partir de ce repiquage est de l'ordre de 100 p. 100 pour les autres traitements ayant passé l'été 1976 en pépinière $\left(C_{1}, D_{1}\right.$ et $\left.E_{1}\right)$, il semble donc bien confirmé qu'une phase de forçage (solution nutritive + serre $20^{\circ} \mathrm{C}$ jours longs) soit nécessaire pour lever définitivement l'inhibition de croissance et pour assurer une résistance suffisante des plants.

La comparaison des traitements $A$ et $B_{1}$ avec le traitement $B_{2}$ montre qu'une éventuelle détérioration du système racinaire par le gel durant l'hivernage n'est pas en 


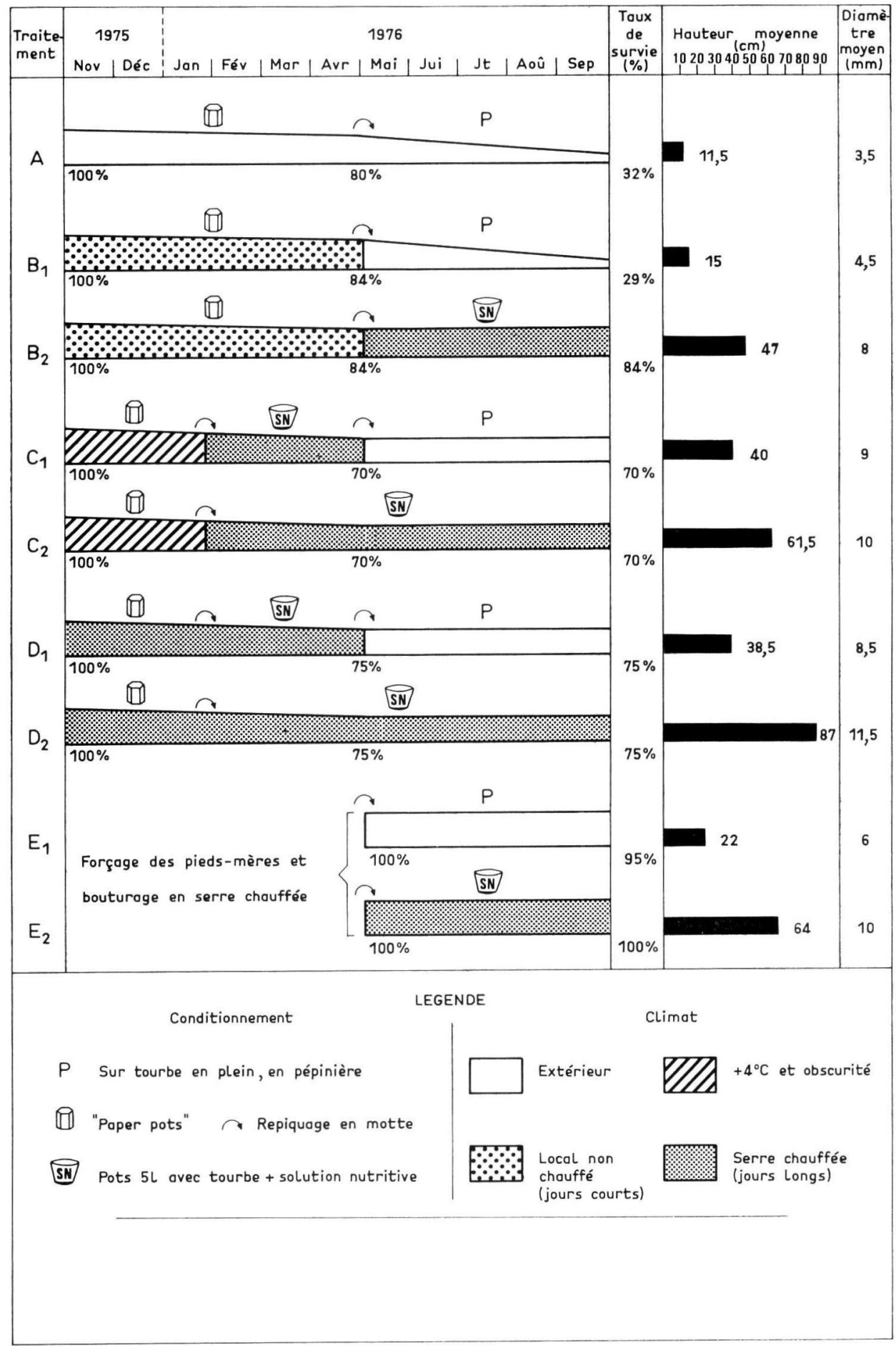

FIG. 1. - Taux de survie, hauteur moyenne ef diamètre moyen au collet des plants obtenus dans les différents traitements.

Percent of living plants, mean height and mean basal diameter of plants in different treatments. 
cause. La comparaison des résultats de tous les traitements montre d'autre part que la hauteur des plants obtenus est d'autant plus grande que le séjour en conditions solution nutritive + serre $20^{\circ} \mathrm{C}$ jours longs s'est prolongé longtemps.

Les plus grands plants (hauteur moyenne 87 centimètres, hauteur maximale 180 centimètres) sont obtenus avec le traitement $D_{2}$, pour lequel les meilleures conditions ont été maintenues pendant toute la durée de l'expérience (et ceci malgré leur débourrement étalé et leur première pousse anormale). Enfin, les traitements $E_{1}$ et $E_{2}$, qui ne comportent pas de phase d'arrêt de croissance, donnent les meilleurs taux de survie.

Une phase de forçage est donc nécessaire pour lever l'inhibition de croissance et obtenir par la suite une croissance normale en pépinière (plants de plus de 40 centimètres de hauteur moyenne en un an, ce qui est comparable à ce qu'on obtient à partir de semis dans les mêmes conditions).

II est possible que cette phase de forçage puisse être raccourcie (elle a durée au minimum 3 mois $1 / 2$ dans nos expériences : traitement $C_{1}$ ). Ce sera l'objet d'expérimentations ultérieures.

Dans de bonnes conditions, nous avons vu que le taux de survie à partir des boutures racinées était toujours supérieur à 70 p. 100 . Comme, d'autre part, il est facile d'obtenir des taux d'enracinement de 80 p. 100, il n'y a pas de difficulté à produire plus de 50 plants à partir de 100 boutures prélevées sur des jeunes plants ou sur des rejets de souche.

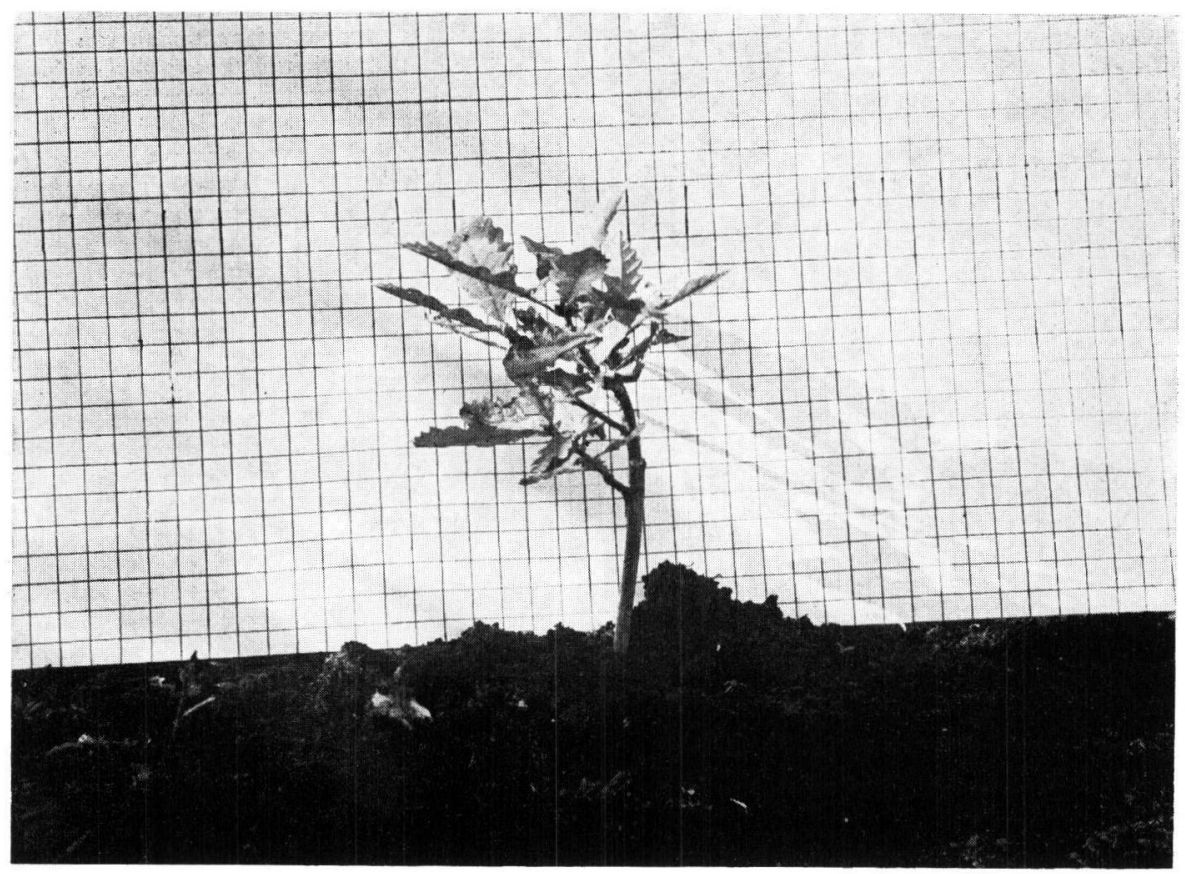

PHOTO 1. - Plant du traitement $B_{1}$ (quadrillage de $1 \mathrm{~cm}$ ).

Plant of $B_{1}$ treatment (squares of $1 \mathrm{~cm}$ ). 
Enfin, le traitement $E_{2}$ montre qu'il est possible de réaliser 3 générations de boutures en 2 ans, à condition de disposer d'une serre chauffée et éclairée en hiver : un plant de 60 centimètres permet en effet le prélèvement de plusieurs boutures ayant une très bonne aptitude à l'enracinement, comme l'a montré un essai annexe.

\section{Morphologie des plants obtenus}

\section{Partie aérienne}

D'une façon générale, la dominance apicale semble faible chez les plants issus de boutures et ceci d'autant plus que la croissance est faible. Dans les plus mauvais traitements, le port est souvent buissonnant (photo 1). Dans les traitements assurant

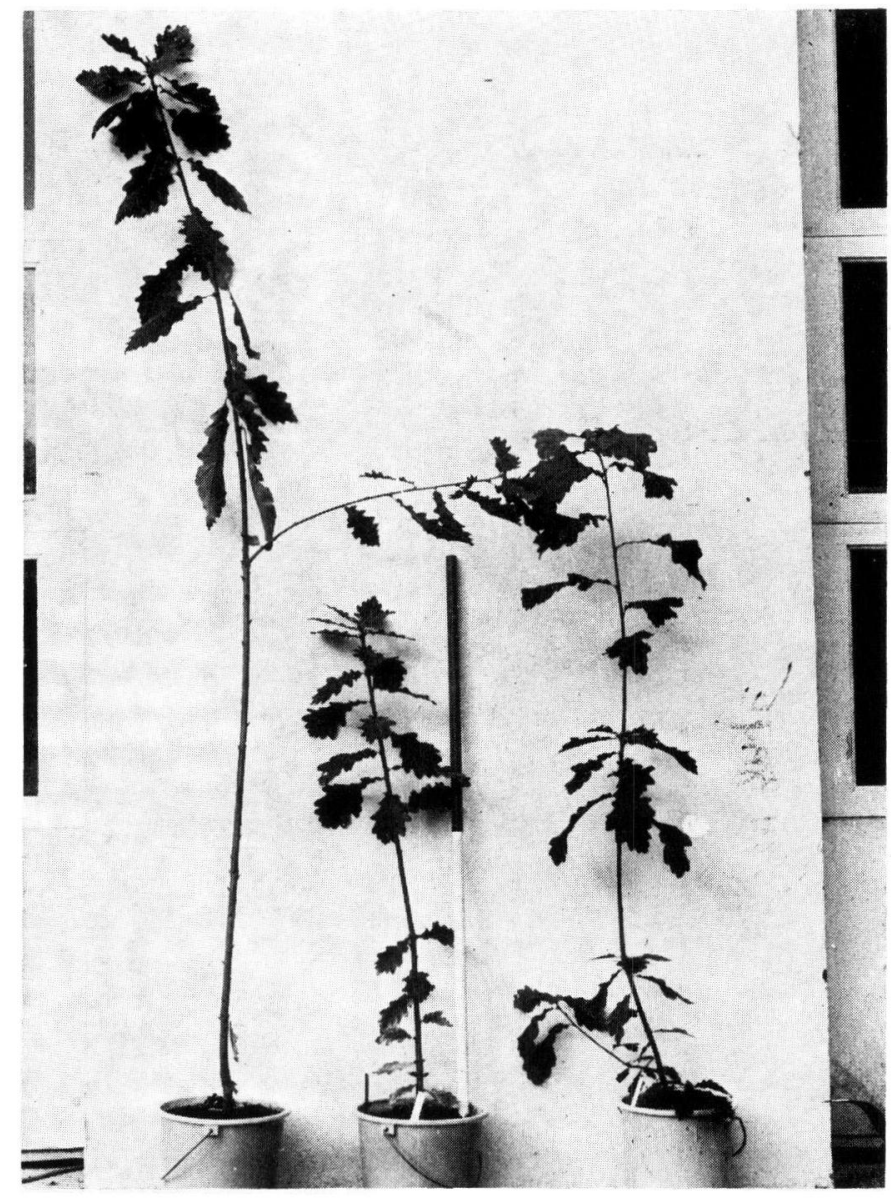

PHOTO 2. - Plants du traitement $D_{2}$ ayant une forme satisfaisante : forte dominance apicale (le bâton fait $1 \mathrm{~m}$ de long).

Plants of $D_{2}$ treatment with a good shape : strong apical dominance (the stick is $1 \mathrm{~m}$ long). 
une forte croissance, la pousse terminale est le plus souvent très vigoureuse (photo 2), ce qui n'empêche pas parfois le développement de pousses basales plagiotropes de grande longueur (photo 3). Dans les cas extrêmes (rares), la pousse terminale est inexistante ou complètement dominée par les pousses basales plagiotropes (photo 4).

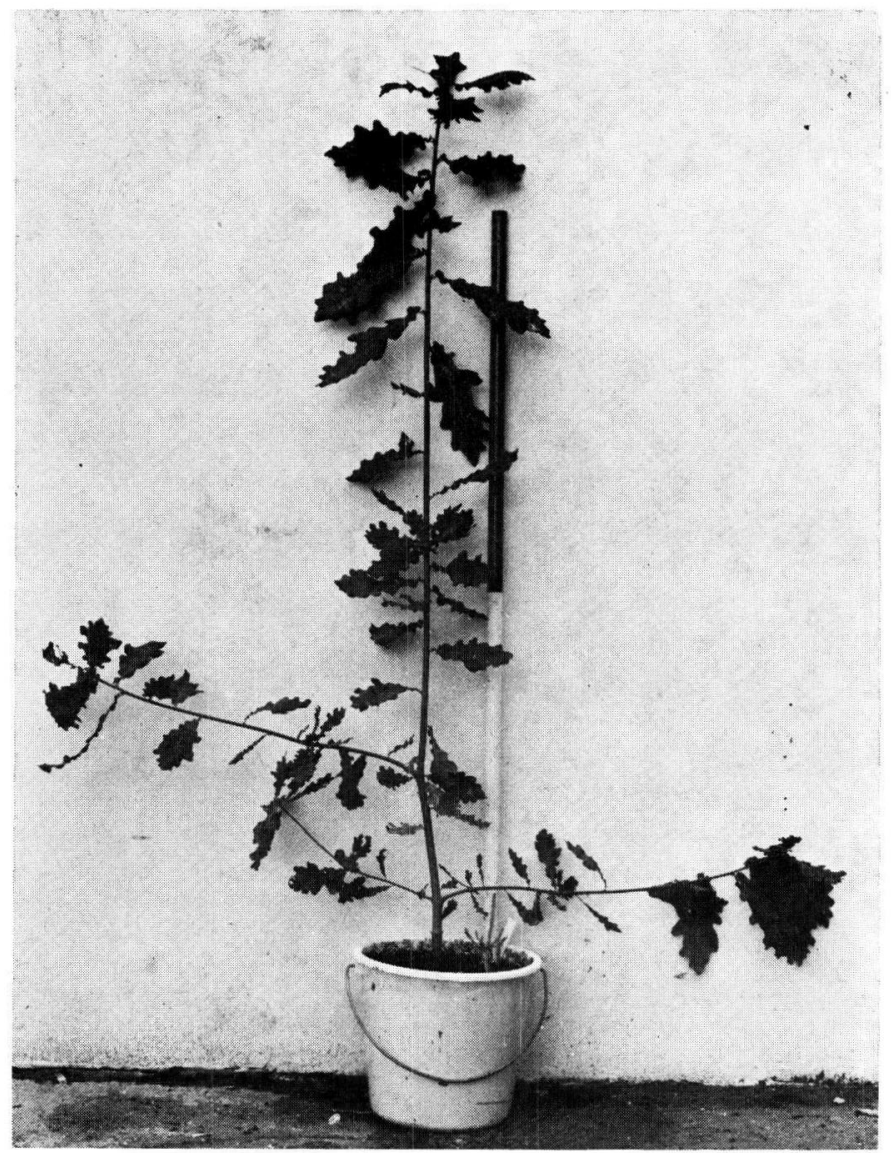

PHOTO 3. - Plant du traitement $D_{2}$ ayant une forte dominance apicale, mais de fortes branches basales plagiotropes (le bâton fait $1 \mathrm{~m}$ de long).

Plant from $D_{2}$ treatment with a strong apical dominance,

but strong horizontal branches in the lower part (the stick is $1 \mathrm{~m}$ long).

De nouvelles expériences et des observations suivies seront nécessaires pour savoir si les défauts de forme observés sur certains plants sont définitifs et s'il est possible d'y remédier. Il semble cependant qu'une forte croissance réduise les risques.

Les premiers essais de taille et d'élagage que nous avons effectués montrent que ces procédés peuvent remédier, semble-t-il, à ces défauts de forme. 


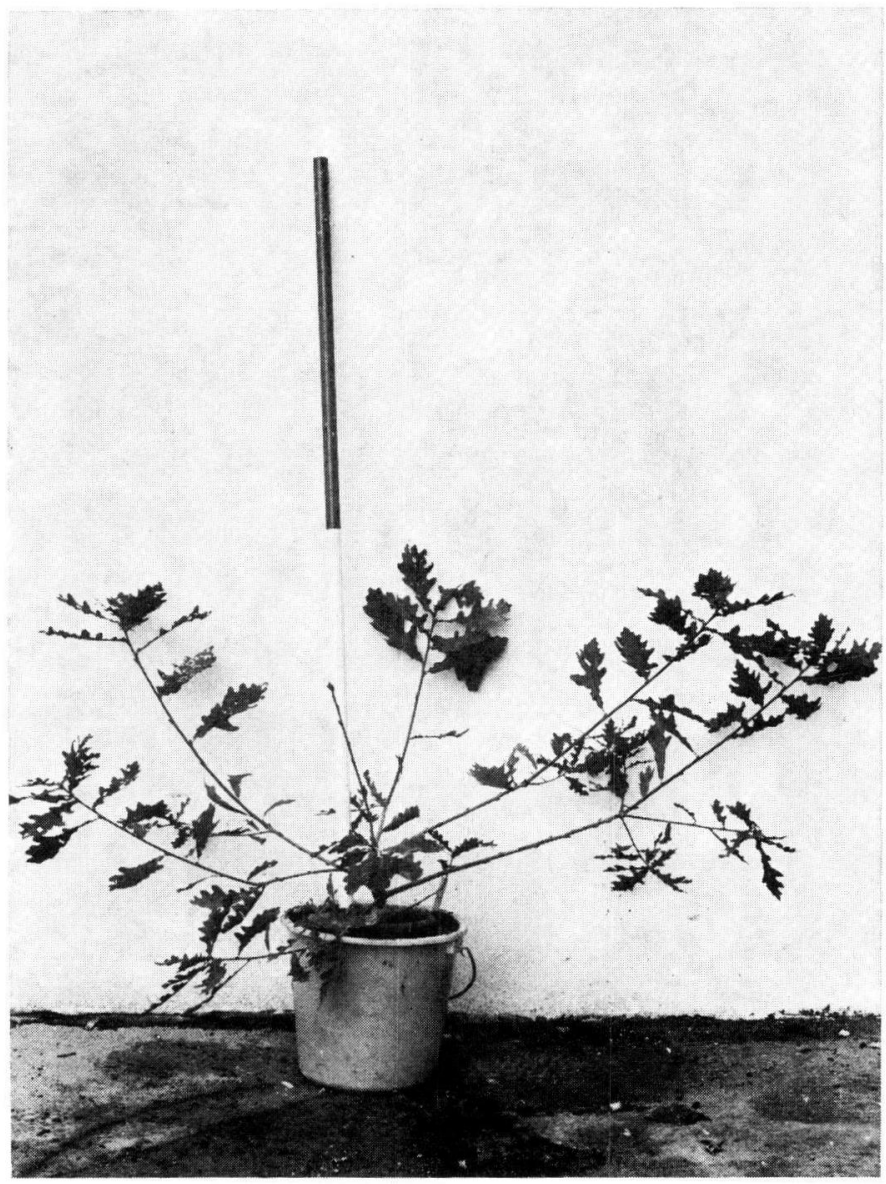

PHOTO 4. - Plant du traitement $D_{2}$ n'ayant pas de dominance apicale (le bâton fait $1 \mathrm{~m}$ de long). Plant from $D_{2}$ treatment without any apical dominance (the stick is $1 \mathrm{~m}$ long).

\section{Systèmes racinaires}

Cette première expérience ayant été essentiellement réalisée en containers pendant au moins une partie de la vie des plants, les déformations racinaires sont évidemment importantes (racines spiralées, «chignons»), et il n'est pas possible de se prononcer sur la « qualité » de l'enracinement au sens du reboiseur. Cependant, certaines observations ont une portée générale :

- mis à part leur direction de croissance, les racines des plants issus de boutures présentent le même aspect que des racines de semis (ramification, chevelu, écorce) ;

- lors du bouturage, les jeunes racines issues de la base de la bouture poussent horizontalement (Cornu, Garbaye, Le Tacon, 1976). Cependant, au bout d'un an, les plants présentent au moins une racine plongeante de plus fort diamètre que les 
autres (oblique, sinon parfaitement verticale) qui ressemble beaucoup au pivot d'un plant issu de semis.

Ceci est vrai indépendamment des déformations éventuellement dues au pot (photos 5, 6 et 7). Cette tendance est d'autant plus marquée que le plant est vigoureux (traitements $B_{2}, C_{2}, D_{2}$ ). Ces observations sont à rapprocher des derniers travaux concernant la morphogenèse du système racinaire du semis de chêne (Riedacker, 1976 ; communication orale) : si l'on freine le développement d'un pivot issu de gland, une racine latérale qui normclement serait restée fine, courte et plagiotrope, peut en prendre le relais et atteindre une dimension comparable à celle qu'avait eu le pivot unique. Il semblerait même qu'elle puisse devenir oblique tout comme les grosses racines des boutures. Une certaine analogie apparaît donc entre les racines latérales issues de pivots et les racines issues de boutures. En attendant des résultats expérimentaux précis dans ce domaine, il semble donc qu'un enracinement

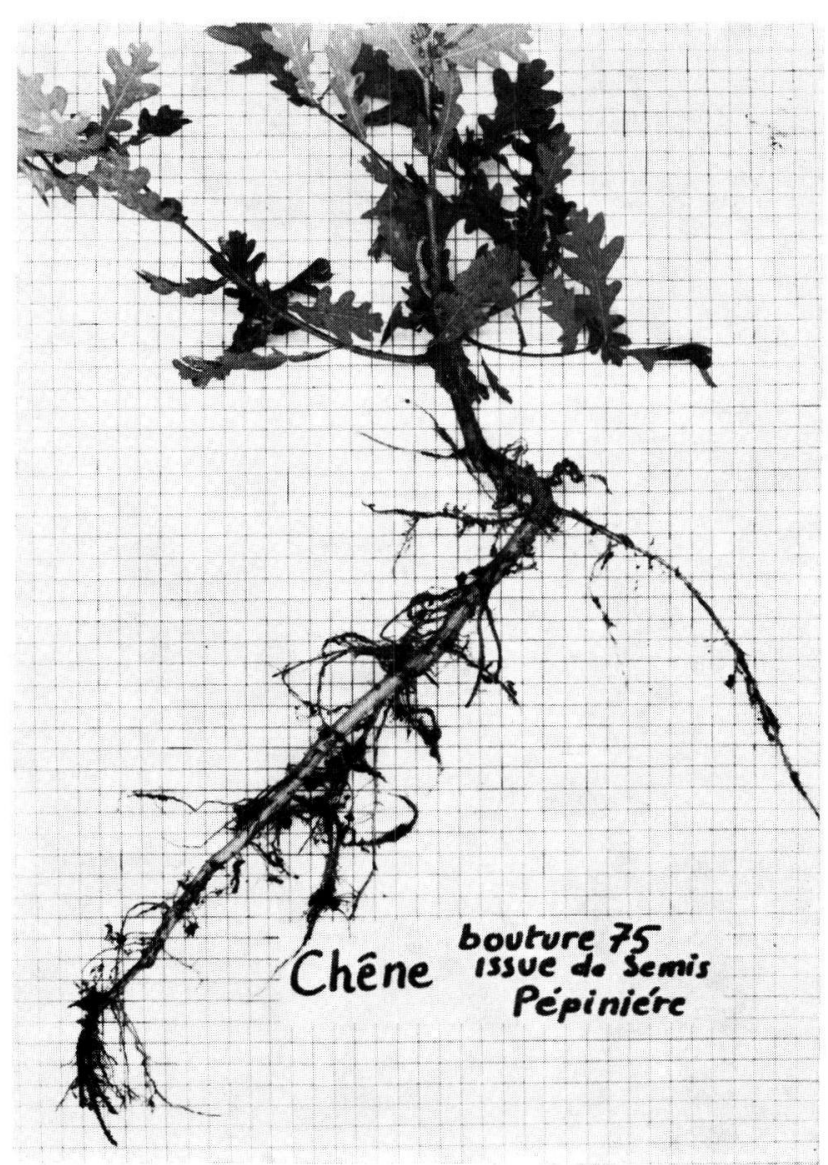

PHOTO 5. - Système racinaire d'un plant du traitement $B_{1}$ (quadrillage de $1 \mathrm{~cm}$ ). Root system of a plant from $B_{1}$ treatment (squares of $1 \mathrm{~cm}$ ). 


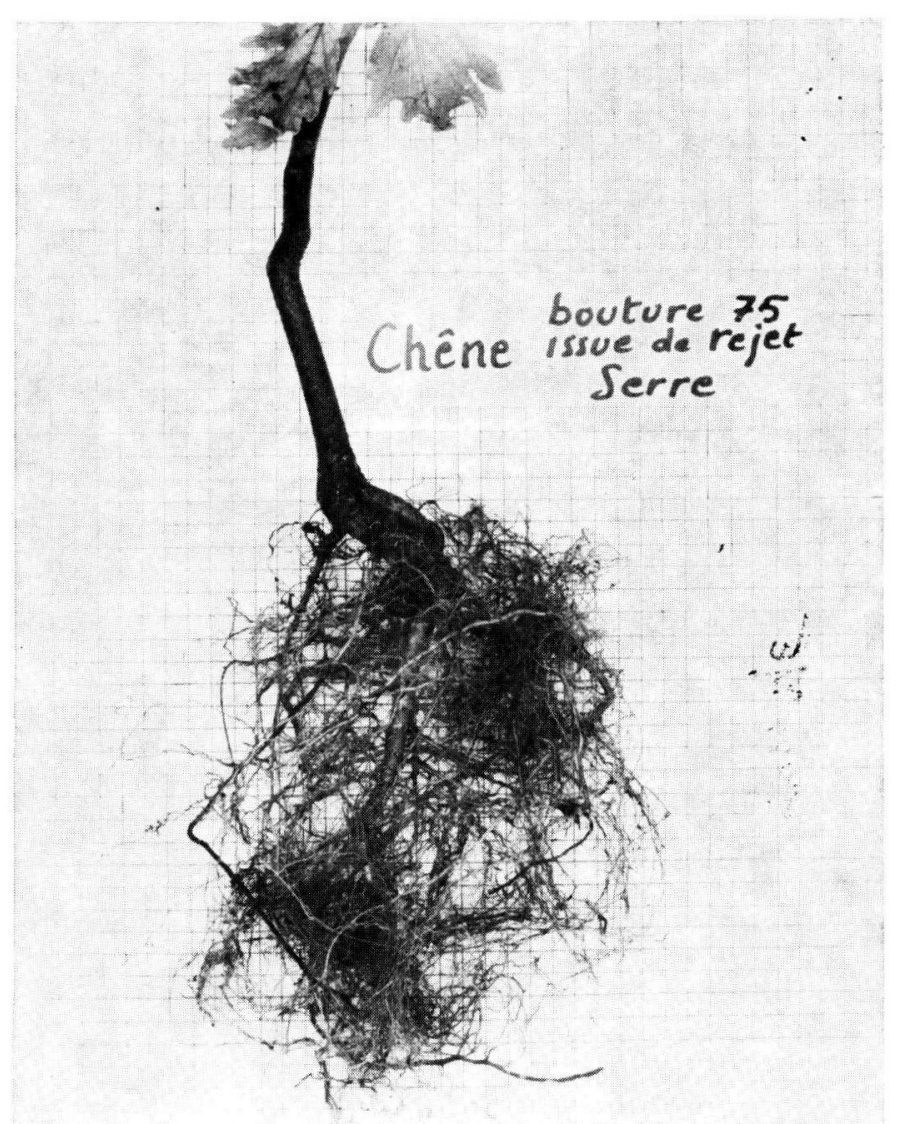

PHOTO 6. - Système racinaire d'un plant du traitement $C_{2}$ (quadrillage de $1 \mathrm{~cm}$ ). Root system of a plant from $C_{2}$ treatment (squares of $1 \mathrm{~cm}$ ).

se rapprochant beaucoup de celui d'un semis puisse être obtenu à condition de réaliser les meilleures conditions de croissance possible au début de la vie du plant.

On remarque, par ailleurs, que le caractère plagiotrope des racines et l'inhibition de croissance de la partie aérienne semblent liés. Ce phénomène, que l'on retrouve chez le hêtre (Cornu, Garbaye, Le Tacon, 1976), ne s'observe pas chez des espèces qui se bouturent beaucoup plus facilement (frêne, érable, merisier). Chez ces espèces l'enracinement est beaucoup plus rapide dans les mêmes conditions et beaucoup plus abondant, avec des racines moins plagiotropes.

Nous avons donc de bonnes raisons de penser que l'inhibition est définitivement levée chez les plants de chêne qui ont présenté une forte croissance et une disparition de la plagiotropie des racines. II est cependant trop tôt pour proposer une explication de ces faits; notre but était avant tout de mettre au point une technique.

Nous pouvons aussi penser qu'avec certaines souches de chêne particulièrement aptes au bouturage, et dans des conditions optimales (date de prélèvement, matériel très jeune, etc.) un enracinement rapide, puissant et non plagiotrope pourrait être 


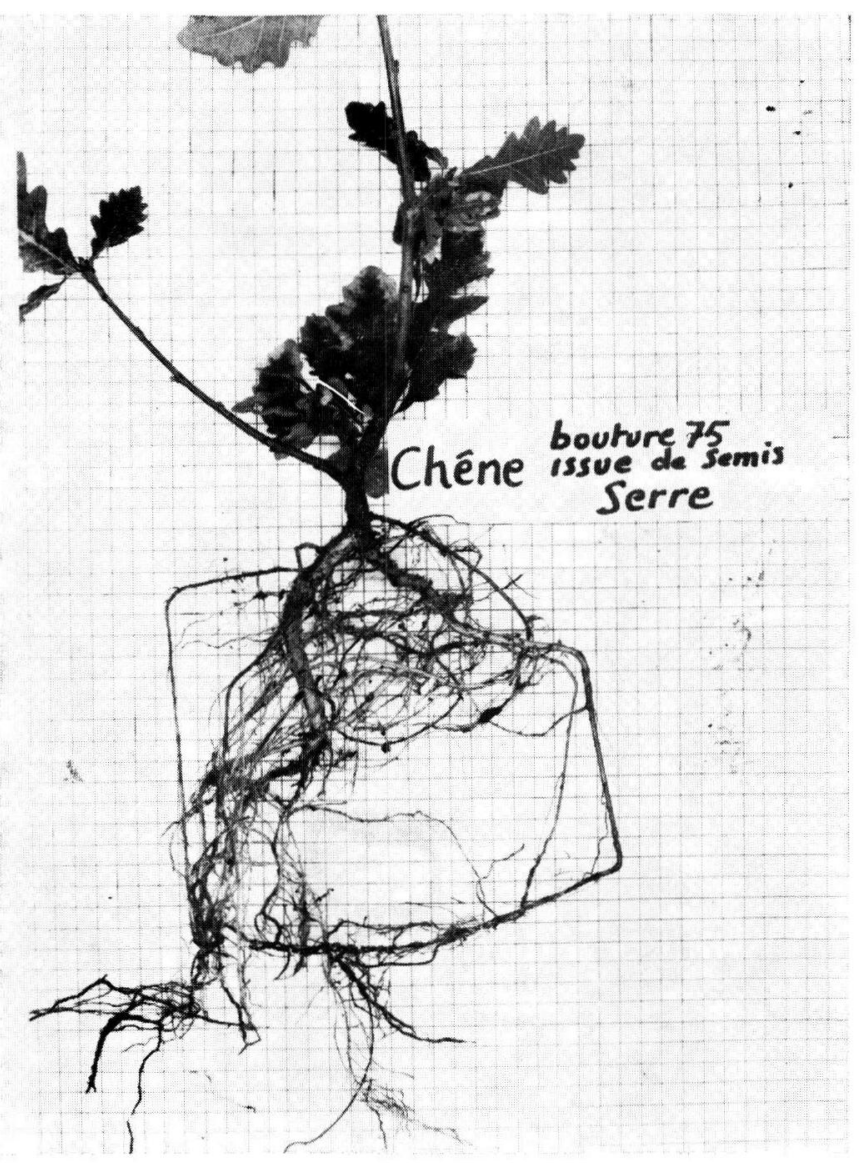

PHOTO 7. - Système racinaire d'un plant du tr aitement $D_{2}$ (quadrillage de $1 \mathrm{~cm}$ ). Root system of a plant from $D_{2}$ treat ment (squares of $1 \mathrm{~cm}$ ).

directement obtenu. Dans ces conditions, il est probable que les phénomènes d'inhibition ne se manifesteraient pas.

L'observation de la base de la bouture initiale montre que lorsque celle-ci est encore visible, elle est souvent le siège de pourritures. Ce n'est que chez les plants les plus vigoureux que la cicatrisation est parfaite (photo no 8 ).

Ici encore, une croissance rapide du jeune plant est donc souhaitable puisqu'elle supprime une porte d'entrée possible aux parasites lors de la plantation.

\section{Conclusions pratiques}

Au terme de cette première expérimentation, nous sommes en mesure de tirer quelques conclusions pratiques concernant la production rapide de plants de chêne à partir de boutures enracinées : 


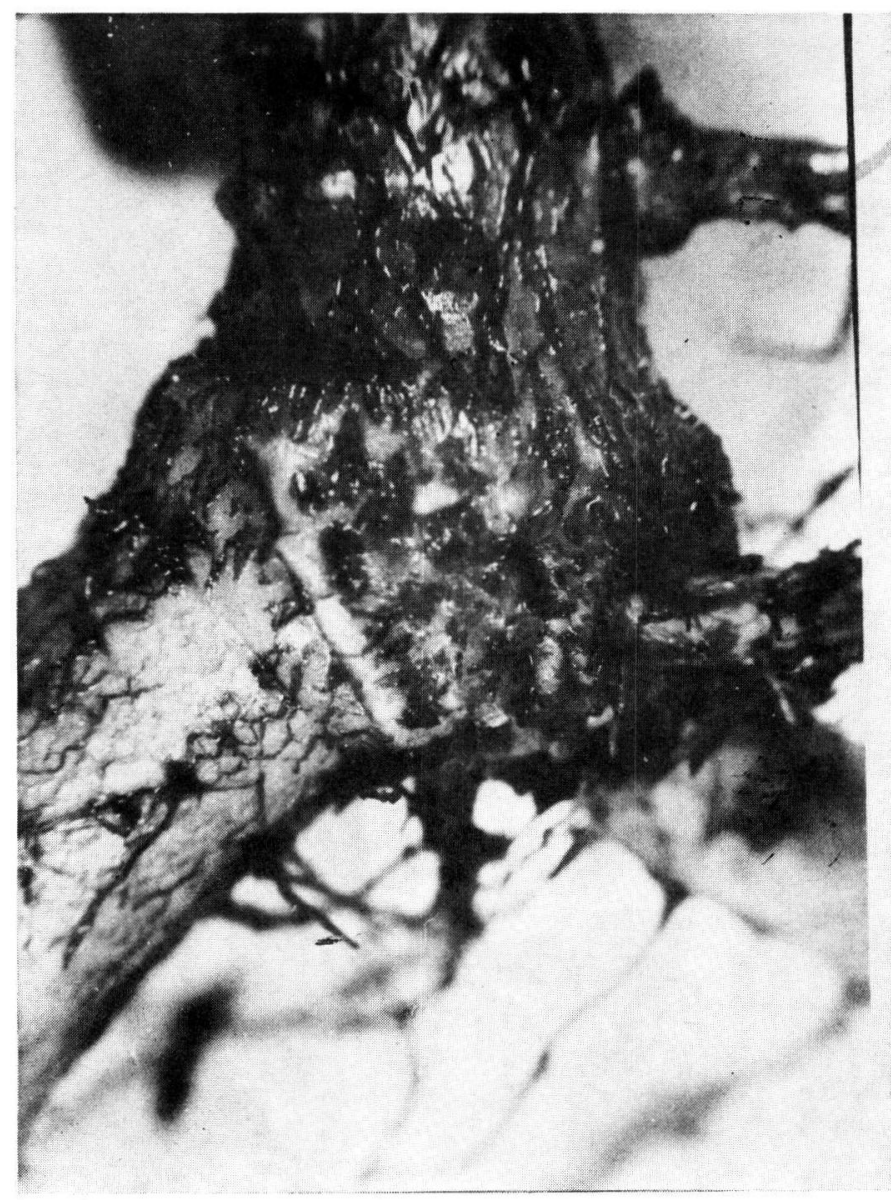

PHOTO 8. - Cicatrisation totale de la base de la bouture.

Total cicatrisation of the end of the cutting.

- Dans des conditions de culture adéquates, le potentiel de croissance des boutures est tout à fait comparable à celui des semis.

- Dans les conditions où nous avons pratiqué le bouturage, les jeunes boutures enracinées semblent présenter une inhibition de croissance que l'on peut lever par un forçage de quelques mois en serre chauffée avec jours longs et solution nutritive. Les plants peuvent ensuite terminer leur croissance en pépinière dans des conditions normales. La technique de culture sur tourbe fertilisée prend alors tout son intérêt (Delran, Garbaye, Le Tacon, 1975).

- Une période de froid préalable à la phase de forçage n'est pas indispensable, mais améliore l'homogénéité de la reprise de croissance.

- La morphologie des plants obtenus se rapproche d'autant plus de celle des semis (port de la partie aérienne avec dominance apicale, racines plongeantes) que la croissance est rapide. 
- Pour multiplier rapidement un clone à des fins expérimentales, il est possible de réaliser 3 générations de boutures en 2 ans à condition de disposer d'une serre chauffée et éclairée en hiver.

Ces quelques résultats, qui restent à expliquer par des expérimentations plus fines, posent donc les bases techniques nécessaires à la multiplication végétative du chêne.

Reçu pour publication en février 1977.

\title{
Summary
}

Development of rooted cuttings of oak

(Quercus petraea (M.) Liebl.). First elements of a

plant-production technic

\begin{abstract}
Vegetative multiplication of sessile oak is possible by mist propagation. The rooted cuttings show a plagiotropic root system and an inhibition of the development of aerial parts after a period of no-growth conditions.

This inhibition can be suppressed by a period of some months (minimal length not determined) in optimal growth conditions : long days, day temperature over $20^{\circ} \mathrm{C}$ and watering with nutritive solution on peat medium.

Then, the plants can resume their growth in the nursery in normal conditions, and can reach in one year the same height as seedlings.

A cold period before optimal growth conditions is not necessary, but it improves the homogeneity of the growth start.

The faster is the growth, the more similar is the morphology of the plants to the one of seedlings (shape of the aerial part, diving roots).
\end{abstract}

\section{Zusammenfassung}

\author{
Waschstum der bewurzelten eichenstecklingen \\ (Quercus petraea (M.) Liebl.). Erste grundlagen \\ eines techniks für forstpflanzenproduktion
}

Die vegetative Vermehrung von $Q$. petraea durch Stecklinge junger Triebe unter " mist " ist möglich. Die bewurzelten Stecklinge besitzen ein plagiotropisches Wurzelsystem und eine Hemmung des Wachstums des überirdischen Teils nach einer Phase des Stillstands der Vegetation.

Diese Hemmung kann durch eine Periode von einigen. Monaten (Minimaldauer noch unbestimmt) unter optimalen Wachstumsbedingungen (Langtage, Temperaturen am Tage über $20^{\circ} \mathrm{C}$, Ernährung mittels Lösung auf Torfsubstrat) behoben werden.

Die Pflänzlinge können dann ihr Wachstum in der Baumschule unter normalen Bedingungen beenden und innerhalb eines Jahres die gleiche Grösse wie Sämlinge erreichen. Eine der Treibphase vorhergehende Kälteperiode ist nicht unerlässlich, verbessert jedoch eine einheitlicheWiederaufnahme des Wachstums.

Die Morphologie der so erhaltenen Pflanzen nähert sich desto mehr derjenigen der Sämlinge (Wuchs des überirdischen Teils, Wurzeltiefgang) als das Wachstum rasch vor sich geht.

\section{Références bibliographiques}

COIC Y., LESAINT C., 1973. La nutrition minérale en horticulture avancée. La Revue horticole, aoûtsept., no 2316 (29-34).

CORNU D., GARBAYE J., LE TACON F., 1975. Résultats d'un essai préliminaire sur le bouturage du chêne et du hêtre. R.F.F. XXVII, 2, 75 (139-140). 
CORNU D., DELRAN S., GARBAYE J., LE TACON F., 1977. Recherche des meilleures conditions d'enracinement des boutures herbacées de chêne rouvre et de hêtre. Ann. Sci. forest., vol. 34, no 1, 1977.

DELRAN S., GARBAYE J., LE TACON F., 1976. Production rapide de plants feuillus sur tourbe fertilisée. R.F.F., XXVII, 6.

KLEINSCHMITT J., WITTE R., SAUER A., 1975. Möglichkeiten der züchterischen Verbesserung von Stiel und- Traubeneichen. II : Versuche zur Stecklingsvermehrung von Eiche. Allg. Forst. u. J. Ztg., 146, Jg. 10. 